\title{
Acrolein is formed from trienoic fatty acids in chloroplast: $A$ targeted metabolomics approach
}

\author{
Jun'ichi Mano ${ }^{1,2, *}$, Sergey Khorobrykh ${ }^{1,3}$, Kenji Matsui ${ }^{4}$, Yoko lijima $^{5, a}$, \\ Nozomu Sakurai ${ }^{5}$, Hideyuki Suzuki ${ }^{5}$, Daisuke Shibata ${ }^{5}$ \\ ${ }^{1}$ Science Research Center, Yamaguchi University, Yoshida 1677-1, Yamaguchi 753-8515, Japan; ${ }^{2}$ Graduate School of \\ Agriculture, Yamaguchi University, Yoshida 1677-1, Yamaguchi 753-8515, Japan; ${ }^{3}$ Molecular Plant Biology, Department of \\ Biochemistry, University of Turku, FI-20014 Turku, Finland; ${ }^{4}$ Graduate School of Medicine, Yamaguchi University, Yoshida \\ 1677-1, Yamaguchi 753-8515, Japan; ${ }^{5}$ Kazusa DNA Institute, Kazusa Kamatari 2-6-7, Kisaradzu 292-0818, Japan \\ *E-mail: mano@yamaguchi-u.ac.jp Tel: +81-83-933-5945 Fax: +81-83-933-5944
}

Received September 14, 2014; accepted November 12, 2014 (Edited by T. Koeduka)

\begin{abstract}
Aldehydes and ketones produced from lipid peroxides (oxylipin carbonyls) exhibit a variety of biological activities ranging from the induction of defense genes to the irreversible damage to cells. Short oxylipin carbonyls such as acrolein and (E)-2-pentenal are responsible for tissue injury under environmental stress, but their production mechanism remains unclear. In this study, we elucidated the source fatty acids of short oxylipin carbonyls in leaves. We first established a comprehensive analysis of oxylipin carbonyls for quantitation and structural estimation. Carbonyls were extracted from rosette leaves of Arabidopsis thaliana, derivatized with 2,4-dinitrophenylhydrazine and separated with a reverse-phase HPLC equipped with a photodiode array detector and an Fourier transform ion cyclotron resonance mass spectrometer. Thirty-three distinct carbonyls were detected, in which 19 species were identified on the MS/MS spectrum. Using this analysis system, we compared the carbonyl composition in the leaves between two A. thaliana lines that have different fatty acid composition. The mutant $f a d 7 f a d 8$, which lacks the biosynthesis of trienoic fatty acids in the plastid, contained significantly lower amounts of malondialdehyde, acrolein and (E)-2-pentenal and higher amounts of acetone, 3-pentanone, and $n$-hexanal than the wild type Col-0. This difference in the carbonyl composition agreed with the oxidative degradation of dienoic and trienoic fatty acids in vitro, showing that similar non-enzymatic reactions occur in the thylakoid membrane. Consideration of the formation mechanism of acrolein from trienoic fatty acid suggests that membrane lipids in chloroplasts are constitutively oxidized by singlet oxygen.
\end{abstract}

Key words: Lipid peroxide, oxidative stress, polyunsaturated fatty acid, reactive carbonyl species.

Production of reactive oxygen species (ROS) such as superoxide anion radical and hydrogen peroxide is intrinsically associated with intracellular redox reactions. In plants, ROS are constitutively produced via the electron leakage from the electron transport chains in chloroplasts and mitochondria (Asada 2006) and via oxidase reactions in peroxisomes. The concentrations of ROS in cells are kept low levels by the abundant antioxidant molecules and enzymes. When cells receive internal stimuli such as hormones or external ones such as the changes in environmental conditions, the ROS levels are transiently increased to trigger physiological responses (Mittler et al. 2011). When the antioxidant capacity is decreased by severe stress, ROS levels rise higher and longer so that ROS oxidize various biomolecules rather indiscriminately, leading to damages to cells (Mano et al. 2002). The function of ROS to trigger various physiological responses in plants is broadly appreciated. There is a keen interest in biochemical processes of this oxidative signaling (Møller and Sweetlove 2010).

Increasing evidence shows that the lipid peroxide $(\mathrm{LOOH})$-derived aldehydes and ketones (oxylipin carbonyls) are endogenous effectors of oxidative signal (Farmer and Mueller 2013; Mano 2012). ROS are formed constitutively in or on the thylakoid membrane and the mitochondrial inner membrane (Asada 2006). ROS are also formed transiently on the surface of the plasma membranes, due to the stimulus-induced activation of respiratory burst oxidase-homologous NADPH oxidases (Foreman et al. 2003). Membrane lipids are the most immediate and abundant targets of

Abbreviations: DNP-, 2,4-dinitrophenylhydrazo-; DNPH, 2,4-dinitrophenylhydrazine; HHE, 4-hydroxy-(E)-2-hexenal; HNE, 4-hydroxy-(E)-2nonenal; $\mathrm{LOOH}$, lipid peroxide; MDA, malondialdehyde.

a Present address: Faculty of Applied Bioscience, Kanagawa Institute of Technology, 1030 Shimo-Ogino, Atsugi, Kanagawa 243-0292, Japan

This article can be found at http://www.jspcmb.jp/

Published online December 25, 2014 
the ROS thus generated and are inevitably oxidized to LOOHs (Mène-Saffrané et al. 2007). LOOHs decompose spontaneously or are degraded enzymatically to produce an array of oxylipin carbonyls with various carbon chain length, number and position of double bonds and those of hydroxyl- or oxo-group addition (Blée 1998). Oxylipin carbonyls exert signaling function or toxicity, depending on their levels (Mano et al. 2005; Matsui 2006; Mueller et al. 2008; Taki et al. 2005; Weber et al. 2004). The biological activities of these compounds are largely ascribed to the chemical reactivity of the carbonyl moiety. In addition, the oxylipin carbonyls comprising the $\alpha, \beta$-unsaturated bond (reactive carbonyls; RCS) have high electrophilicity and can form covalent modification on proteins and nucleic acids (Esterbauer et al. 1991), and cause tissue injury in plants under environmental stress (Mano 2012).

In plants, more than a dozen species of oxylipin carbonyl have been found (Yamauchi et al. 2012 and reviewed by Mano et al. 2009; Yin et al. 2010). Because each oxylipin carbonyl has distinct chemical reactivity and hence toxicity (Mano et al. 2009; Reynolds 1977), it is important to identify the chemial species and determine the amount of every carbonyl generated in the cells. To obtain detailed insights into the oxylipin carbonyl metabolism in plants, we previously established a HPLC analytical method (Matsui et al. 2009). In this method, carbonyls extracted from plants are derivatized with 2,4-dinitrophenylhydrazine (DNPH), the resulting hydrazone are extracted, separated on a reverse-phase chromatography and determined with a ultraviolet detector. Using this method, we revealed that plants produce oxylipin carbonyls including RCS constitutively under non-stress conditions, and the levels of some of those carbonyls are increased on stress, as follows. For example, in the leaves after photoinhibitory illumination, acrolein and (E)-2-pentenal were increased. The overexpression of 2-alkenal reductase, an enzyme to detoxify RCS (Mano et al. 2002), suppressed their increase and reduced leaf injury (Mano et al. 2010). In aluminium-stressed roots, following RCS were increased on stress and suppressed by the overexpression of 2-alkenal reductase; acrolein, malondialdehyde (MDA), 4-hydroxy-(E)-2-hexenal (HHE), 4-hydroxy-(E)-2nonenal (HNE) and (E)-2-heptenal. In addition to these, several saturated carbonyls such as formaldehyde, acetaldehyde, $n$-hexanal and $n$-octanal showed similar changes (Yin et al. 2010). These short chain (C9 or less) carbonyls appear to be responsible for the tissue injury. The production of C6 and C9 carbonyls can be ascribed to the hydroperoxide lyase reactions on $\mathrm{LOOHs}$ (Gardner and Hamberg 1993; Matsui 2006), but the production mechanism of other carbonyls are largely unknown. We have particular interest in acrolein among these oxylipin carbonyls because it is the most highly electrophilic and hence most toxic oxylipin carbonyl (Esterbauer et al. 1991). In this study, we analyzed carbonyls in a fatty acid desaturase-mutant of Arabidopsis thaliana and determined the source fatty acids of carbonyls.

\section{Meterials and methods}

\section{Plant materials}

Seeds of $f a d 7 f a d 8$ were kind gift from Prof. Ko Iba, Kyushu University. Plants were grown on soil at $23^{\circ} \mathrm{C}$ in a $12 \mathrm{~h} / 12 \mathrm{~h}$ photoperiod at $50 \mu \mathrm{mol}$ photons $\mathrm{m}^{-2} \mathrm{~s}^{-1}$ with white fluorescent lamps.

\section{Chemicals}

Acetonitrile of an HPLC grade, 3,3-diethoxy-1-propene (acrolein acetal) and 1,1,3,3-tetramethoxypropane (MDA acetal) were obtained from Wako Pure Chemical Industries, Ltd. (Tokyo, Japan). HNE acetal were from Alexis Japan care of Biolinks (Tokyo). HHE was obtained from Cayman Chemical (Ann Arbor, MI, USA). Other carbonyl compounds were of reagent grade. DNPH (containing 50\% water; Wako) was purified by recrystallization in acetonitrile and dissolved in the same solvent at $20 \mathrm{mM}$, using an absorption coefficient $14,791 \mathrm{M}^{-1} \mathrm{~cm}^{-1}$ at $350 \mathrm{~nm}$.

\section{Preparation of standard DNP-derivatives of authentic carbonyl compounds}

To a DNPH solution ( $0.3 \mathrm{~g}$ in $20 \mathrm{ml}$ ethanol), $1 \mathrm{mmol}$ of aldehyde or ketone was added, followed by a few drops of formic acid. The solution was incubated at $50^{\circ} \mathrm{C}$ with gentle stirring until the color changes, and then kept at ambient temperature overnight. Resulting crystals of the DNP-derivative were collected on a glass filter. For acrolein, MDA and HNE, the corresponding acetal was first dissolved at $1-10 \mathrm{mM}$ in $0.1 \mathrm{M} \mathrm{HCl}$, and incubated at $40^{\circ} \mathrm{C}$ for $1 \mathrm{~h}$. DNPH $(0.035 \%$ in $1 \mathrm{M} \mathrm{HCl})$ was added to an equimolar amount, and was incubated at ambient temperature for $1 \mathrm{~h}$. The DNP-derivative was extracted to hexane, crystallized by chilling and collected. The molar quantity of a DNP-derivative was determined by weight.

\section{LC-FTICR-MS analysis}

Carbonyls were extracted from rosette leaves with acetonitrile, derivatized with $\mathrm{DNPH}$, and the resulting derivatives were extracted as previously reported (Mano et al. 2010; Yin et al. 2010). An Agilent 1100 system (Agilent, http://www.home.agilent.com) coupled with a Finnigan LTQ-FT (Thermo Electron, Rockford, IL, USA) was used for liquid chromatography-Fourier transform ion cyclotron resonance mass spectrometer (FTICR-MS) analysis as previously described (Iijima et al. 2008). The data were acquired and browsed using Xcalibur software version 2.0 (Thermo Electron, Rockford, IL, USA). DNP-derivatized sample in acetonitrile was applied to 
a Wakosil DNPH-II column $(4.6 \times 150 \mathrm{~mm}$; Wako Pure Chemical Industries, Ltd., http://www.wako-chem. co.jp/english/). Flow rate was set to $0.6 \mathrm{ml} \mathrm{min}^{-1}$, with column temperature at $40^{\circ} \mathrm{C}$ and the sample volume $20 \mu \mathrm{l}$ to inject. The DNPH-II Eluents A and B (Wako Pure Chemical Industries, Ltd.) were used as the mobile phase, with the following gradient program: $0-5 \mathrm{~min}, 100 \% \mathrm{~A}$; 5-30 min, linear gradient from $100 \%$ A to $100 \% \mathrm{~B}, 30-$ $60 \mathrm{~min}, 100 \% \mathrm{~B}$, and then $60.1-68 \mathrm{~min} 100 \% \mathrm{~A}$ for reequilibration. To monitor LC elution, a photodiode array detector was used in the wavelength range $250-450 \mathrm{~nm}$. The APCI setting was as follows: APCI negative mode; source voltage $6 \mathrm{kV}$; capillary temp, $275^{\circ} \mathrm{C}$; capillary voltage, $-6 \mathrm{kV}$; vaporizer temp, $450^{\circ} \mathrm{C}$; sheath gas flow, 50; and aux gas flow, 5. Ions of high intensity detected in the primary FTICR-MS were further analyzed with the secondary ion trap MS with collision energy 35\%. Atomic composition of an unidentified compound was deduced from the $\mathrm{m} / \mathrm{z}$ value using Xcalibur software.

\section{Analysis of fatty acid composition}

Lipids were extracted from rosette leaves $(0.10$ to $0.28 \mathrm{~g}$ ) of $A$. thaliana, hydrolyzed and methylated in $\mathrm{HCl}-$ acidic methanol with a fixed amount of heptadecanoic acid as the internal standard, and the resulting fatty acid methyl esters were extracted, as previously described (Takami et al. 2010). The methyl ester mixture was separated and determined with a gas chromatography- mass spectrometer (QP2010 Plus, Shimadzu, Kyoto, Japan) equipped with a DB-Wax column (30 m length $\times 0.25 \mathrm{~mm}$ i.d., Agilent Technologies Japan, Hachioji, Japan). The temperatures of the injector and interface were 240 and $200^{\circ} \mathrm{C}$, respectively. Injection was performed using a split-mode with a ratio of 100 . The column temperature was $180^{\circ} \mathrm{C}$. The carrier gas $(\mathrm{He})$ was delivered at $86.1 \mathrm{kPa}$. Mass detection range was $\mathrm{m} / \mathrm{z} 40-$ 350. The amount of methyl-esters was estimated from the signal intensity of the total ion.

\section{Results}

\section{Aldehydes and ketones are distiguinshable}

In our previous studies, identified carbonyls occupied only half a member of the detected peaks of putative carbonyls. Attempting to obtain structural information of detected carbonyls, we here employed a FTICR-MS as a detector.

Both aldehydes and ketones react with DNPH to form the hydrazones (dinitrophenylhydrazo (DNP)derivatives). We prepared DNP derivatives of 24 types of carbonyls of 1-10 carbon chain length with or without an unsaturated bond (Table 1). In the MS/MS spectra of all these derivatives but that of malondialdehyde (MDA), the fragment ion of $\mathrm{m} / \mathrm{z} 179$, which is formed via the rearrangement of the DNP moiety (Kölliker et al. 2001), was included within the ten most intensive signals

Table 1. List of authentic carbonyls and their conversion factors with a single wavelength detector (at $340 \mathrm{~nm}$ ) and a photodiode array detector (integration from $300-400 \mathrm{~nm}$ ).

\begin{tabular}{|c|c|c|c|c|c|}
\hline \multirow{2}{*}{$\mathrm{C} n$} & \multirow{2}{*}{ Aldehyde/Ketone } & \multirow{2}{*}{$\mathrm{C}=\mathrm{C}$ bond } & \multirow{2}{*}{ Carbonyl } & \multicolumn{2}{|c|}{ Conversion factor } \\
\hline & & & & $340 \mathrm{~nm}$ & $\Sigma(300-400 \mathrm{~nm})$ \\
\hline 1 & A & 0 & formaldehyde & 3.32 & 4.23 \\
\hline 2 & A & 0 & acetaldehyde & 0.49 & 0.52 \\
\hline \multirow[t]{4}{*}{3} & A & 0 & propionaldehyde & 0.56 & 0.60 \\
\hline & A & 1 & acrolein & 1.36 & 1.12 \\
\hline & $\mathrm{A}$ & 0 & MDA & 3.24 & 11.66 \\
\hline & $\mathrm{K}$ & 0 & acetone & 3.66 & 3.43 \\
\hline \multirow[t]{4}{*}{4} & A & 0 & butyraldehyde & 0.54 & 0.94 \\
\hline & A & 1 & crotonaldehyde & 2.89 & 1.95 \\
\hline & A & 1 & methacrolein & 2.04 & 1.62 \\
\hline & $\mathrm{K}$ & 0 & 2-butanone & 1.61 & 1.47 \\
\hline \multirow[t]{3}{*}{5} & A & 0 & $n$-pentanal & 0.62 & 0.63 \\
\hline & A & 1 & (E)-2-pentenal & 2.71 & 1.82 \\
\hline & $\mathrm{K}$ & 0 & 3-pentanone & 1.90 & 1.71 \\
\hline \multirow[t]{4}{*}{6} & A & 0 & $n$-hexanal & 0.46 & 0.46 \\
\hline & A & 1 & (E)-2-hexenal & 2.36 & 2.78 \\
\hline & A & 1 & (Z)-3-hexenal & 0.52 & 0.53 \\
\hline & A & 1 & HHE & 0.61 & 0.76 \\
\hline 7 & A & 0 & $n$-heptanal & 0.64 & 0.66 \\
\hline \multirow[t]{3}{*}{8} & A & 0 & $n$-octanal & 0.89 & 0.90 \\
\hline & A & 3 & phenylacetaldehyde & 0.59 & 0.62 \\
\hline & A & 0 & 2-ethyl-hexanal & 1.00 & 1.00 \\
\hline 9 & A & 0 & $n$-nonanal & 1.53 & 1.60 \\
\hline \multirow[t]{2}{*}{10} & A & 0 & decanal & 1.10 & 1.12 \\
\hline & A & 1 & decenal & 3.58 & 2.34 \\
\hline
\end{tabular}


(Supplemental data. Table S1). The m/z 179 fragment ion is thus a robust marker of a DNP derivative of a carbonyl. MDA, a dicarbonyl, undergoes the two-step reaction with DNPH and forms a cyclic derivative (Andreoli et al. 2003), which does not generate the m/z 179 fragment ion. The DNP-MDA derivative is identified on the retention time of $\mathrm{LC}$ and the $\mathrm{m} / \mathrm{z}$ value of the precursor ion.

We found that all the DNP-aldehyde derivatives (except for the DNP-MDA) generated the $\mathrm{m} / \mathrm{z} 163$ signal within the six most intensive signals in their MS/ MS spectra (Supplemental data. Table S1). This signal is typical to the DNP-aldehyde derivative (Andreoli et al. 2003) and is presumed to represent the cyclic $\mathrm{C}_{7} \mathrm{H}_{5} \mathrm{~N}_{3} \mathrm{O}_{2}$ product of the rearrangement of the fragment containing the $\mathrm{C}$ atom and a $\mathrm{H}$ atom of aldehyde (Zweiner et al. 2002). In the DNP-derivative of a ketone, the $\mathrm{H}$ is substituted with an alkyl group and therefore the fragment of this size will not be generated (Supplemental data. Figure S1). Indeed, all the DNP-derivatives of four ketones (acetone, 2-butanone, 2-petanone, 3-pentanone) did not generate the $\mathrm{m} / \mathrm{z} 163$ fragment in the 10 most intensive signals. Thus the $\mathrm{m} / \mathrm{z} 163$ fragment can be employed as an aldehyde-specific marker. If the MS/MS spectrum of a carbonyl does not contain this fragment within 6 most intensive signals, then the carbonyl is regarded as a ketone.

\section{Quantitation of carbonyls on the ultraviolet absorbance}

We employed the absorbance chromatogram for quantifying carbonyls because their DNP-carbonyl derivatives show relatively strong ultraviolet absorption. DNP-carbonyl derivatives have absorption maximum in the wavelength range of $350-380 \mathrm{~nm}$, but the DNP-MDA derivative shows the maximum at $307 \mathrm{~nm}$ (Fenaille et al. 2001). In our previous method using a single wavelength detector, we set the detection wavelength at $340 \mathrm{~nm}$ to detect MDA and other carbonyls at the same time, with a sacrifice of the sensitivity (Matsui et al. 2009). The current apparatus is equipped with a photodiode array detector. To cover a broad range of carbonyls and to increase the sensitivity, we integrated the absorption from 300 to $400 \mathrm{~nm}$.

The content of a carbonyl in a plant sample is calculated from the peak area (300-400 nm integration) of the DNP derivative of the carbonyl. Because the efficiencies of the DNP derivatization and extraction of the derivative, and the molar absorption coefficient differ by carbonyls, we obtained the conversion coefficient for each carbonyl (Table 1). The molar absorption coefficients of the DNP derivatives of C1-C7 carbonyls, either saturated or unsaturated, fall into a narrow range (18.5-25.8 $\times 10^{3} \mathrm{M}^{-1} \mathrm{~cm}^{-1}$ ) (Pötter and Karst 1996), but the conversion coefficients of these carbonyls varied broader. This variation therefore reflects the differences of the efficiency of DNP derivatization and the extraction of the derivative.

To verify whether the peaks in the absorption chromatogram (300-400 nm) of plant samples represent DNP derivatives, we compared them with peaks in the selected ion monitoring (SIM) chromatogram of the

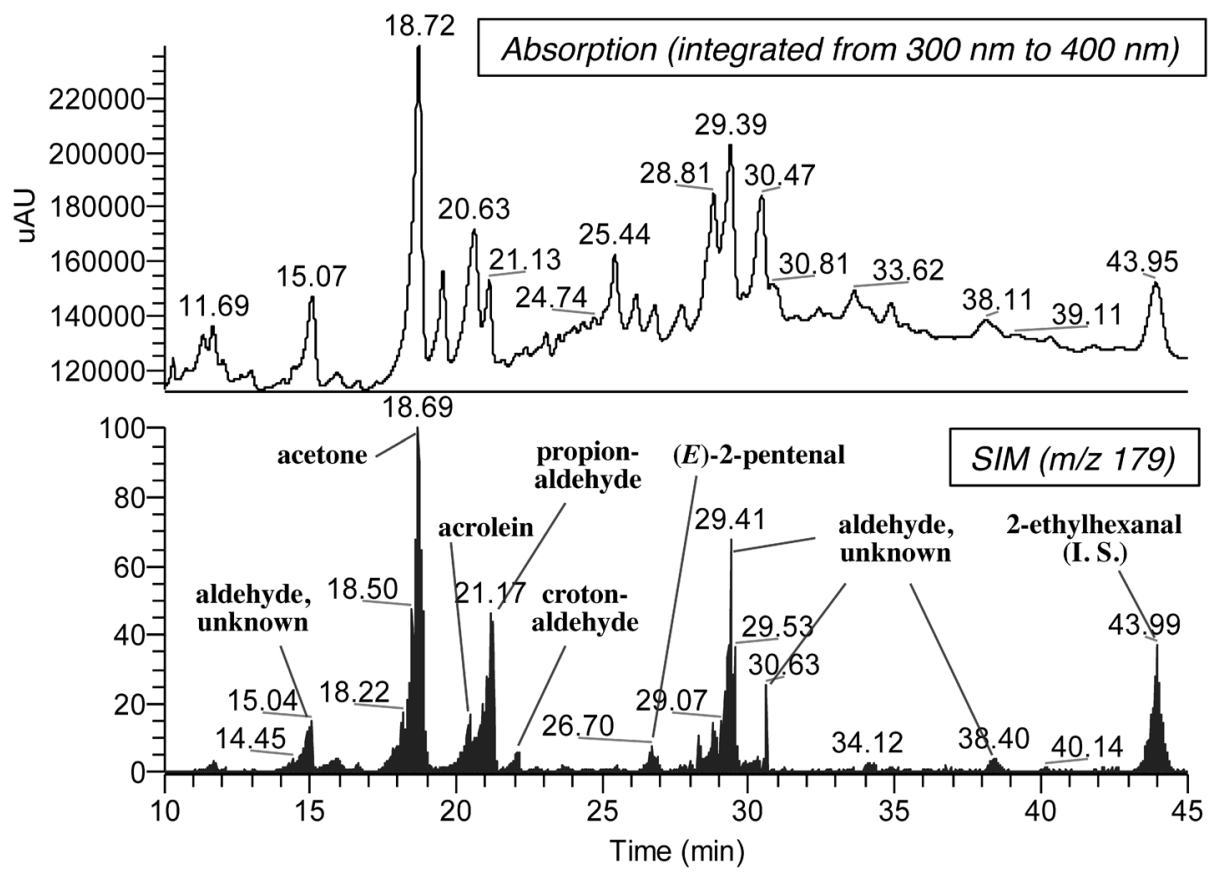

Figure 1. Comparison of absorption chromatogram (top) and the SIM chromatogram (bottom) of the DNP-carbonyls prepared from A. thaliana Col-0 leaf extract. 
DNP derivative marker m/z 179 fragment (Figure 1). The peaks in both chromatograms matched. This assures that DNP-carbonyls in plant samples can be specifically detected by absorption. False detection of other compounds such as pigments is negligible.

\section{Annotation of unidentified carbonyls}

We identified 19 kinds of carbonyls in A. thaliana Col-0 leaves (Figure 2). The identity of these carbonyls were confirmed on their retention time and the MS/MS spectra as compared with those parameters obtained for authentic compounds (data not shown). The leaves also contained as many unidentified carbonyl peaks as identified. Many unidentified carbonyls were also detected in tobacco tissues previously (Mano et al. 2010; Yin et al. 2010). We here attempted to estimate the structure of them in the following way. (i) From the high resolution MS data of the pseudo-molecular ion [M$\mathrm{H}]^{-}$in the negative ion mode, we deduced the atomic composition of a DNP-carbonyl, and then obtained that of the original carbonyl. (ii) On this carbonyl's atomic composition, we retrieved compounds in a public databases of organic compounds, i.e., PubChem (http:// pubchem.ncbi.nlm.nih.gov) and The Chemical Entities of Biological Interest (http://www.ebi.ac.uk/chebi/). (iii) From the obtained compounds, we manually selected compounds that are composed of only $\mathrm{C}, \mathrm{H}$, and $\mathrm{O}$ atoms and contain a carbonyl structure(s). These compounds are the candidates. From these candidates, we further picked up the compounds that are reported to occur in organisms or to be an lipid oxidation product. Of the 14 unidentified carbonyls in A. thaliana leaves, only one carbonyl of $\mathrm{C}_{2} \mathrm{H}_{4} \mathrm{O}_{2}$ was unequivocally annotated as glycolaldehyde (Table 2).

\section{Differential carbonyl contents in two A. thaliana lines of different fatty acid composition}

To obtain insights into the production mechanism of oxylipin carbonyls, we compared the carbonyl composition of two A. thaliana lines with different fatty

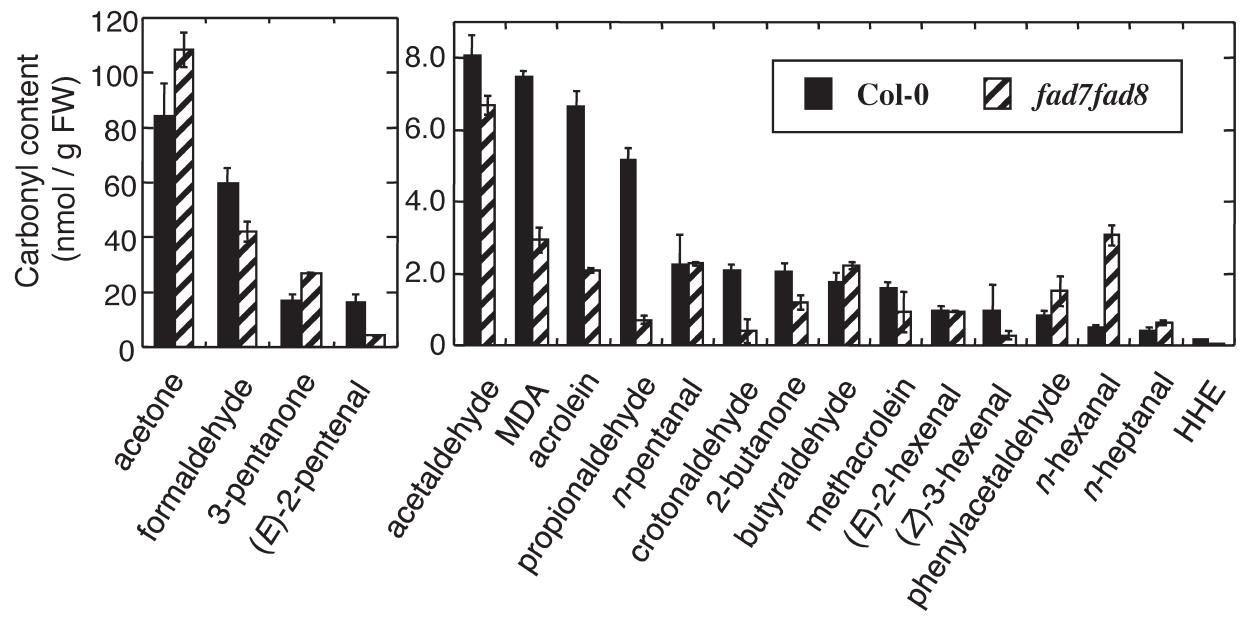

Figure 2. Carbonyl contents in leaves of A. thaliana Col-0 (black) and fad7fad8 (hatched). Carbonyl contents were determined from the absorbance chromatograms. Error bars indicate the standard deviation among plants $(n=3)$.

Table 2. Annotation of unidentified carbonyls.

\begin{tabular}{|c|c|c|c|c|c|}
\hline $\begin{array}{l}\text { Relative } \\
\text { retention } \\
\text { time }\end{array}$ & $\begin{array}{l}\text { Deduced } \\
\text { molecular } \\
\text { weight }\end{array}$ & $\begin{array}{l}\text { Molecular } \\
\text { formula }\end{array}$ & $\begin{array}{l}\text { Aldehyde or } \\
\text { Ketone }\end{array}$ & $\begin{array}{l}\text { Number of } \\
\text { candidate } \\
\text { carbonyls }\end{array}$ & Annotation \\
\hline 0.088 & 238.052 & $\mathrm{C}_{15} \mathrm{H}_{10} \mathrm{O}_{3}$ & $\mathrm{~K}$ & $100<$ & \\
\hline 0.146 & 60.022 & $\mathrm{C}_{2} \mathrm{H}_{4} \mathrm{O}_{2}$ & A & 1 & glycolaldehyde \\
\hline 0.196 & 74.038 & $\mathrm{C}_{3} \mathrm{H}_{6} \mathrm{O}_{2}$ & A & 3 & lactaldehyde, 3-hydroxypropanal, methoxyacetaldehyde, \\
\hline 0.216 & 141.043 & $\mathrm{C}_{7} \mathrm{H}_{9} \mathrm{O}_{3}$ & A & 4 & $\begin{array}{l}\text { 6-oxohexan-2-yl formate, }(Z) \text {-5-methyl-6-oxohex-4- } \\
\text { enoate, }(E) \text {-5-methyl-6-oxohex-3-enoate }\end{array}$ \\
\hline 0.426 & 116.048 & $\mathrm{C}_{5} \mathrm{H}_{8} \mathrm{O}_{3}$ & A & 42 & \\
\hline 0.501 & 236.055 & $\mathrm{C}_{16} \mathrm{H}_{12} \mathrm{O}_{2}$ & K & $100<$ & \\
\hline 0.594 & 168.116 & $\mathrm{C}_{10} \mathrm{H}_{16} \mathrm{O}_{2}$ & A & $100<$ & lilac aldehyde, 3-hydroxycyclocitral \\
\hline 0.594 & 159.069 & $\mathrm{C}_{11} \mathrm{H}_{11} \mathrm{O}$ & A & 0 & \\
\hline 0.606 & 130.100 & $\mathrm{C}_{7} \mathrm{H}_{14} \mathrm{O}_{2}$ & A & 47 & \\
\hline 0.626 & 168.116 & $\mathrm{C}_{10} \mathrm{H}_{16} \mathrm{O}_{2}$ & A & $100<$ & \\
\hline 0.636 & 196.110 & $\mathrm{C}_{11} \mathrm{H}_{16} \mathrm{O}_{3}$ & K & $100<$ & \\
\hline 0.638 & 198.126 & $\mathrm{C}_{11} \mathrm{H}_{18} \mathrm{O}_{3}$ & A & $100<$ & \\
\hline 0.669 & 168.077 & $\mathrm{C}_{9} \mathrm{H}_{12} \mathrm{O}_{3}$ & A & 63 & \\
\hline
\end{tabular}


Table 3. Estimation of the carbonyl concentration in the chloroplast. The chloroplast volume on the chlorophyll base in A. thaliana is assumed to be $26 \mu \mathrm{l}(\mathrm{mg} \mathrm{Chl})^{-1}$ (for spinach; Heldt et al. 1973). The chlorophyll content in rosette leaves of both wild type and $f a d 7 f a d 8$ was $0.75 \mathrm{mg} \mathrm{Chl} \mathrm{(g} \mathrm{FW)}{ }^{-1}$. Asterisks represent significant difference between Col- 0 and $f a d 7 f a d 8$ with $p<0.05(*)$ and $<0.01(* *)$ by Student's $t$-test.

\begin{tabular}{|c|c|c|c|c|c|}
\hline \multirow{2}{*}{$\mathrm{Cn}$} & \multirow{2}{*}{ Carbonyl } & \multirow{2}{*}{$\frac{\Delta(\mathrm{Col}-0-\text { fad } 7 \mathrm{fad} 8)}{\mathrm{nmol}(\mathrm{g} \mathrm{FW})^{-1}}$} & \multirow{2}{*}{ Concn (mM) } & \multicolumn{2}{|c|}{ Source fatty acid } \\
\hline & & & & Trienoic & Dienoic \\
\hline 1 & formaldehyde & $17.6 * *$ & 0.90 & V & \\
\hline 2 & acetaldehyde & $1.4^{*}$ & 0.07 & V & \\
\hline \multirow[t]{4}{*}{3} & propionaldehyde & $4.5^{* *}$ & 0.23 & V & \\
\hline & acrolein & $4.6^{* *}$ & 0.24 & V & \\
\hline & malondialdehyde & $4.5^{* *}$ & 0.23 & V & \\
\hline & acetone & $-24.3^{*}$ & 1.25 & & V \\
\hline \multirow[t]{2}{*}{4} & crotonaldehyde & $1.7 * *$ & 0.09 & V & \\
\hline & 2-butenone & $0.9 * *$ & 0.05 & V & \\
\hline \multirow[t]{2}{*}{5} & (E)-2-pentenal & $12.1 * *$ & 0.62 & V & \\
\hline & 3-pentanone & $-9.8 * *$ & 0.50 & & V \\
\hline \multirow[t]{2}{*}{6} & $n$-hexanal & $-2.6 * *$ & 0.13 & & V \\
\hline & HHE & $0.1 * *$ & 0.01 & V & \\
\hline 7 & $n$-heptanal & $-0.2^{*}$ & 0.01 & & V \\
\hline
\end{tabular}

acid composition. The process of the non-enzymatic oxidation of lipids and fatty acids and the subsequent formation of carbonyls have been extensively studied in food chemistry research, and it is known that linoleic acid and linolenic acid produce different sets of carbonyls. For example, $n$-pentanal and $n$-hexanal are produced preferentially from linoleic acid, while propionaldehyde and (E)-2-pentenal are from linolenic acid (Grosch 1987). We tested if non-enzymatic oxidation operate in leaf cells, using an A. thaliana mutant with altered fatty acid composition: The genes FAD7 and FAD8 encode the plastidial $\omega$-3 fatty acid desaturases that convert dienoic fatty acids, e.g. linoleic acid, to trienoic fatty acids, e.g. linolenic acid, and hence the double-deficient mutant fad7fad8 (McConn et al. 1994), a descendant of the A. thaliana ecotype Columbia (Matsuda et al. 2001), do not synthesize trienoic fatty acids in the plastid. This mutant, in its leaves, contained lower amounts of trienoic fatty acids, i.e., 18:3 and $16: 3(9.6 \%$ and $0 \%$ of the total fatty acids, respectively) and higher amounts of dienoic acids, i.e., 18:2 and $16: 2(57.1 \%$ and $11.0 \%$, respectively) than the wild type $(48.3 \%$ and $10.9 \%$ for $18: 3$ and $16: 3$ versus $17.9 \%$ and $0.8 \%$ for $18: 2$ and $16: 2$ ) under the current growth condition (Supplemental data. Table S2).

The wild type and the mutant plants were cultured under non-stress conditions for 4 weeks. The growth of the fad7fad8 mutant was not different from that of the wild type. Plants of both lines appeared healthy. We determined the carbonyl contents in their rosette leaves (Figure 2). The wild type contained significantly higher amounts of 9 carbonyl species than the fad7fad8 mutant, specifically, formaldehyde, acetaldehyde, propionaldehyde, acrolein, malondialdehyde, crotonaldehyde, 2-butanone, (E)-2-pentenal and 4-hydroxy-(E)-2-hexenal (HHE). The difference of their contents between the wild type and fad7fad 8 is ascribed to the difference in the fatty acid composition. The results suggest that trienoic acids in the chloroplast are converted to these carbonyls (Table 3). On the other hand, acetone, 3 -pentanone and $n$-hexanal were contained less in the wild type than in fad7fad8. This suggests that they were produced from dienoic acids in chloroplasts.

\section{Discussion}

To elucidate the source fatty acids of biologically important short chain oxylipin carbonyls such as acrolein, we here established a comprehensive analysis method for carbonyls and compared the carbonyl contents in two A. thaliana lines with different fatty acid compositions. The double mutant fad7fad8 lacks the two fatty acid desaturase localized in the plastid, and is deficient of the biosynthesis of trienoic fatty acids (18:3 and 16:3) in the organelle. The rosette leaves of this mutant contained significantly lower amounts of trienoic fatty acids and also 9 species of carbonyls, and in contrast, higher amounts of dienoic acids and three species of carbonyls (Table 3). We concluded that the carbonyls that were contained less in the fad7fad8 mutant were preferentially produced from trienoic fatty acids, and those contained more in the mutant were from dienoic fatty acids.

We here found that wild type contained 3 types of RCS at higher amounts than the fad7fad8 mutant, i.e. (E)-2-pentenal, acrolein and crotonaldehyde (Figure 2). This clearly shows that these RCS are preferentially produced from trienoic fatty acids. These RCS contained in the $f a d 7 f a d 8$ mutant at low levels might be formed from trienoic acids outside of chloroplasts, or from other unknown sources.

We further postulated the production mechanism of these RCS. A simplest mechanism of carbonyl formation 


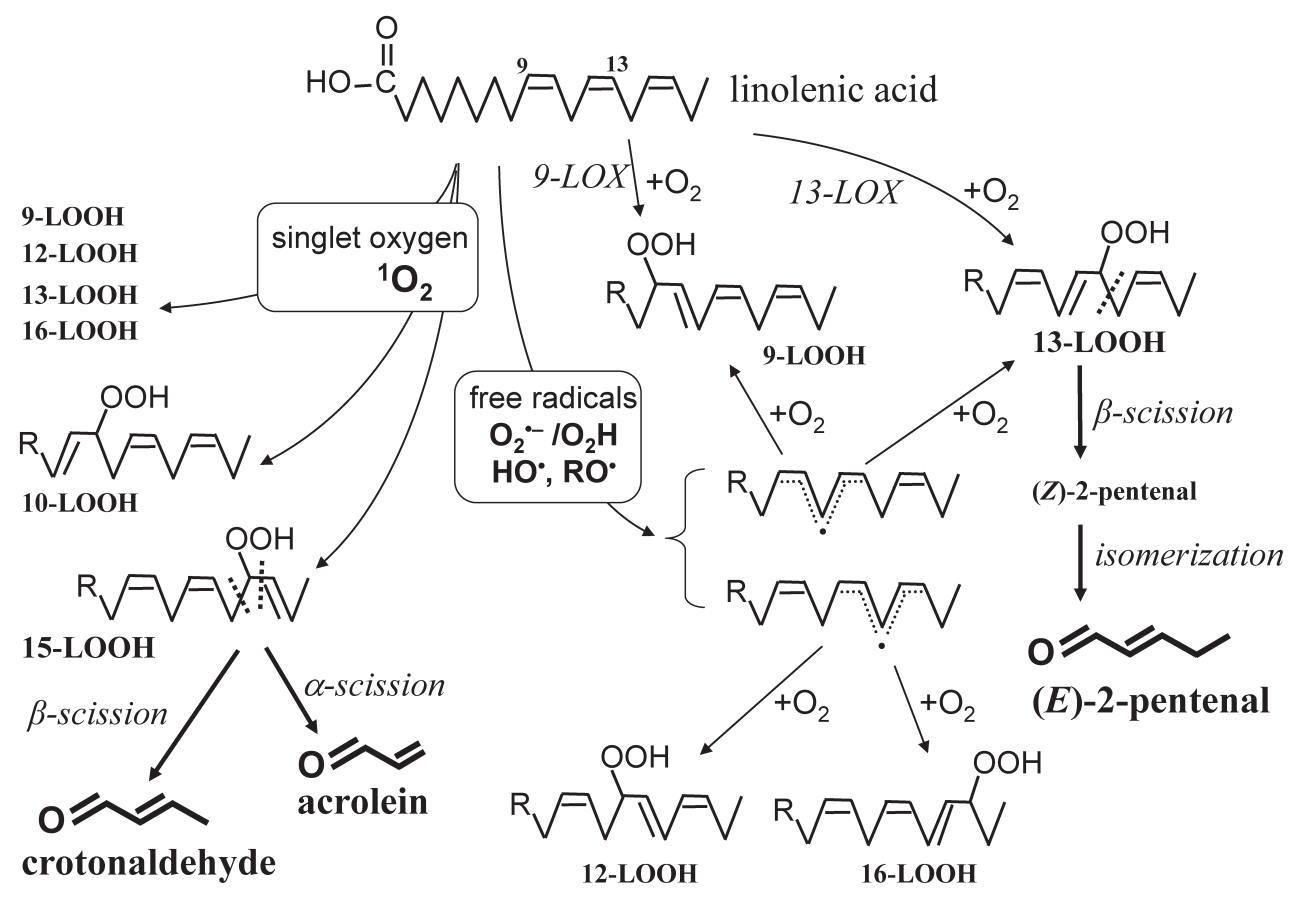

Figure 3. Mechanisms of the formation of acrolein from linolenic acid.

from $\mathrm{LOOH}$ is the homolytic cleavage at the $\mathrm{C}-\mathrm{C}$ bond next to the hydroperoxide. We here assume that the detected oxylipin carbonyls were produced via this onestep reaction (Blée 1998). The formation mechanism of $\mathrm{LOOH}$ is the dioxygenation of an unsaturated fatty acid molecule at either end of a double bond, via the radical-initiated chain reaction or via direct addition of singlet oxygen $\left({ }^{1} \mathrm{O}_{2}\right)$ (Farmer and Mueller 2013). Because acrolein, crotonaldehyde and (E)-2-pentenal were produced preferentially from trienoic fatty acid, their $\alpha, \beta$-unsaturated structure must be derived from the $\omega$-3 double bond of trienoic fatty acids. For acrolein and crotonaldehyde, the source peroxide should be the $15-\mathrm{LOOH}$ of linolenic acid (Figure 3) or the 12-LOOH of hexadecatrienoic acid (for brevity, only the linolenic acid-derived $\mathrm{LOOH}$ will be described below). From other species of $\mathrm{LOOH}$, they are not produced in one step. This in turn suggest that ${ }^{1} \mathrm{O}_{2}$ was involved in the peroxidation of linolenic acid because 15- $\mathrm{LOOH}$ is produced only by the direct addition of ${ }^{1} \mathrm{O}_{2}$, but not by the radicalcatalyzed mechanisms or by lipoxygenases (Figure 3, Montillet et al. 2004). The constitutive formation of acrolein from chloroplastic linolenic acid thus agrees with the constitutive lipid peroxidation by ${ }^{1} \mathrm{O}_{2}$ in chloroplasts (Triantaphylidès et al. 2008; Zoeller et al. 2012). (E)-2-Pentenal, in turn, can be produced from 13$\mathrm{LOOH}$ via its $\beta$-scission and subsequent isomerization.

Among the carbonyls showing different contents between the two lines, MDA, propionaldehyde and (E)-2-pentenal were contained in prominently greater amounts (more than $2.0 \mathrm{nmol}\left(\mathrm{g}\right.$ fresh weight) ${ }^{-1}$ ) in the wild type, and $n$-hexanal and 3 -pentanone was greater in fad7fad8. MDA is thought to be formed via the acid-catalyzed cleavage of bicyclic endoperoxides, which are formed from trienoic fatty acid (Esterbauer et al. 1991). Its preferential occurrence in the wild type suggests the operation of a similar production mechanism in chloroplasts. The preferential production of propionaldehyde and (E)-2-pentenal from trienoic fatty acids and that of $n$-hexanal from dienoic fatty acids agrees with previous results of in vitro analysis of the oxidative degradation products of distinct fatty acids (Grosch 1987).

Interestingly, we found that very short chain carbonyls acetone, formaldehyde and acetaldehyde also showed significant difference in their contents between the two A. thaliana lines (Figure 2, Table 3). As compared with the wild type, the fad7fad 8 leaves contained $29 \%$ greater amounts of acetone, 30\% less formaldehyde and 16\% less acetaldehyde. The production of these carbonyls in plants has been ascribed mainly to alcohol and organic acid metabolisms (Fall 2003), but the present results suggest that the oxidation of fatty acids also significantly contribute to their formation.

From the difference in the content of carbonyls between the wild type and $f a d 7 f a d 8$, we estimated the lowest concentration of each carbonyl in chloroplasts (Table 3). On an assumption that the difference of the content of a carbonyl between the two lines (for acrolein, for example, it was $4.6 \mathrm{nmol}\left(\mathrm{g} \mathrm{FW}^{-1}\right)$ represent its content in the chloroplast, and with the chloroplast volume of $26 \mu \mathrm{l}(\mathrm{mg} \mathrm{Chl})^{-1}$ (for spinach; Heldt et al. 1973), the carbonyl concentration was calculated $(0.24 \mathrm{mM}$ for acrolein, if it is homogeneously 
distributed in the chloroplast). Other trienoic fatty acidderived carbonyls fell into the range from $0.01 \mathrm{mM}$ (for HHE) to $0.90 \mathrm{mM}$ (for formaldehyde). In fad7fad8, the concentration of dienoic fatty acid-derived carbonyls varied from $0.01 \mathrm{mM}$ (for $n$-heptanal) to $0.50 \mathrm{mM}$ (or 3 -pentanone). Because the leaf samples were collected from non-stressed plants, these values represent the basal or physiological levels of carbonyls in the chloroplast. Under oxidative stress conditions, carbonyl levels both in the plastids and in the cells will rise. Indeed, under $\mathrm{NaCl}$ stress conditions, the carbonyl-modification levels of proteins in chloroplasts and in other compartments were increased in A. thaliana leaves (Mano et al. 2014).

Linolenic acid is essential for plants in several aspects. It maintains the membrane fluidity at low temperatures, is the precursor to 12-oxophytodienoic acid and jasmonic acid (McConn et al. 1996), and works as an antioxidant in the membrane (Mène-Saffrané et al. 2009). Our present result shows that linolenic acid generates acrolein and other RCS. Thus the regulation of the saturation/unsaturation of fatty acids in the membrane lipids is critical also for modulating the reactive oxygen and carbonyl species.

\section{Acknowledgements}

The authors are grateful to Yasuhiro Toyota, Hiromi Kumura, Miki Nakashima and Hirotomo Kihara for their excellent technical assistance, Profs. Ko Iba and Osamu Matsuda for kind provision of the fad7fad 8 seeds and Prof. Hiroyuki Ohta for disucussion. This study was supported by Grants-in-Aid for Scientific Research (C) (no. 18570044 to J.M.) and for JSPS Fellows (18-06418 to J.M. and S.K.) by the Japan Society for the Promotion of Science. S.K. stayed in Yamaguchi as a JSPS postdoctral fellow.

\section{References}

Andreoli R, Manini P, Corradi M, Mutti A, Niesen WMA (2003) Determination of patterns of biologically relevant aldehydes in exhaled breath condensate of healthy subjects by liquid chromatography/atmospheric chemical ionization tandem mass spectrometry. Rapid Commun Mass Spectrom 17: 637-645

Asada K (2006) Production and scavenging of reactive oxygen species in chloroplasts and their functions. Plant Physiol 141: 391-396

Blée E (1998) Phytooxylipins and plant defense reactions. Prog Lipid Res 37: 33-72

Esterbauer H, Schaur R, Zollner JH (1991) Chemistry and biochemistry of 4-hydroxynonenal, malonaldehyde and related aldehydes. Free Radic Biol Med 11: 81-128

Fall R (2003) Abundant oxygenates in the atmosphere: a biochemical perspective. Chem Rev 103: 4941-4951

Farmer EE, Mueller MJ (2013) ROS-mediated lipid peroxidation and RES-activated signaling. Annu Rev Plant Biol 64: 429-450

Fenaille F, Mottier P, Turesky RJ, Ali S, Guy PA (2001) Compariton of analytical techniques to quantify malondialdehyde in milk powders. J Chromatogr A 921: 237-245

Foreman J, Demidchik V, Bothwell JHF, Mylona H, Torres MA, Linstead P, Costa S, Brownlee C, Jones JDG, Davies JM, et al.
(2003) Reactive oxygen species produced by NADPH oxidase regulate plant cell growth. Nature 422: 442-446

Gardner HW, Hamberg M (1993) Oxygenation of (3Z)-nonenal to (2E)-4-hydroxy-2-nonenal in the broad bean (Vicia faba L.). J Biol Chem 268: 6971-6977

Grosch W (1987) Reactions of hydroperoxides-Products of low molecular weight. In: Chan HW-S (ed) Autoxidation of Unsaturated Lipids. Academic Press, London, pp 95-139

Heldt HW, Werden K, Milovancev M, Geller G (1973) Alkalization of the chloroplast stroma caused by light-dependent proton flux into the thylakoid space. Biochim Biophys Acta 314: 224-241

Iijima Y, Nakamura Y, Ogata Y, Tanaka K, Sakurai N, Suda K, Suzuki T, Suzuki H, Okazaki K, Kitanaya M, et al. (2008) Metabolite annotations based on the integration of mass spectral information. Plant J 54: 949-962

Kölliker S, Oehme M, Merz L (2001) Unusual MSn fragmentation patterns of 2,4-dinitrophenylhydrazone and its propanone derivative. Rapid Commun Mass Spectrom 15: 2117-2126

Mano J (2002) Early events in environmental stresses in plantsInduction mechanisms of oxidative stress. In: Inzé D,Van Montagu M (eds) Oxidative Stress in Plants. Taylor \& Francis Group, London, pp 217-245

Mano J, Torii Y, Hayashi S, Takimoto K, Matsui K, Nakamura K, Inzé D, Babiychuk E, Kushnir S, Asada K (2002) The NADPH:quinone oxidoreductase P1- $\zeta$-crystallin in Arabidopsis catalyzes the $\alpha, \beta$-hydrogenation of 2 -alkenals: detoxication of the lipid peroxide-derived reactive aldehydes. Plant Cell Physiol 23: 1445-1455

Mano J, Belles-Boix E, Babiychuk E, Inzé D, Torii Y, Hiraoka E, Takimoto K, Slooten L, Asada K, Kushnir S (2005) Protection against photooxidative injury of tobacco leaves by 2 -alkenal reductase. Detoxication of lipid peroxide-derived reactive carbonyls. Plant Physiol 139: 1773-1783

Mano J, Miyatake F, Hiraoka E, Tamoi M (2009) Evaluation of the toxicity of stress-related aldehydes to photosynthesis in chloroplasts. Planta 230: 639-648

Mano J, Tokushige K, Mizoguchi H, Fujii H, Khorobrykh S (2010) Accumulation of lipid peroxide-derived, toxic $\alpha, \beta$-unsaturated aldehydes (E)-2-pentenal, acrolein and (E)-2-hexenal in leaves under photoinhibitory illumination. Plant Biotechnol 27: 193-197

Mano J (2012) Reactive carbonyl species: their production from lipid peroxides, action in environmental stress, and the detoxification mechanism. Plant Physiol Biochem 59: 90-97

Mano J, Nagata M, Okamura S, Shiraya T, Mitsui T (2014) Identification of oxidatively modified proteins in salt-stressed Arabidopsis: A carbonyl-targeted proteomics approach. Plant Cell Physiol 55: 1233-1244

Matsuda O, Watanabe C, Iba K (2001) Hormonal regulation of tissue-specific ectopic expression of an Arabidopsis endoplasmic reticulum-type $\omega$-3 fatty acid desaturate (FAD3) gene. Planta 213: $833-840$

Matsui K (2006) Green leaf volatiles: hydroperoxide lyase pathway of oxylipin metabolism. Curr Opin Plant Biol 9: 274-280

Matsui K, Sugimoto K, Kakumyan P, Khorobrykh SA, Mano J (2009) Volatile oxylipins formed under stress in plants. In: Armstrong D (ed) Lipidomics, Methods in Molecular Biology vol. 580. Humana Press, Totowa, pp 17-27

McConn M, Hugly S, Browse J, Somerville C (1994) A mutation at the fad 8 locus of Arabidopsis identifies a second chloroplast $\omega-3$ desaturase. Plant Physiol 106: 1609-1614

McConn M, Browse J (1996) The critical requirement for linolenic 
acid is pollen development, not photosynthesis, in an Arabidopsis mutant. Plant Cell 8: 403-416

Mène-Saffrané L, Davoine C, Stolz S, Majcherczyk P, Farmer EE (2007) Genetic removal of tri-unsaturated fatty acids suppresses developmental and molecular phenotypes of an Arabidopsis tocopherol-deficient mutant. J Biol Chem 282: 35749-35756

Mène-Saffrané L, Dubugnon L, Chételat A, Stoltz S, GouhierDarimont C, Farmer EE (2009) Nonenzymatic oxidation of trienoic fatty acids contributes to reactive oxygen species management in Arabidopsis. J Biol Chem 284: 1702-1708

Mittler R, Vanderauwera S, Suzuki N, Miller G, Tgnetti VB, Vedepoele K, Gollery M, Shulaev V, Van Breusegem F (2011) ROS signaling: the new wave? Trends Plant Sci 16: 300-309

Montillet J-L, Cacas J-L, Garnier L, Montané M-H, Douki T, Bessoule J-J, Polkowska-Kowalczyk L, Maciejewska U, Agnel J-P, Vial A, et al. (2004) The upstream oxylipin profile of Arabidopsis thaliana: a tool to scan for oxidative stresses. Plant J 40: 439-451

Møller IM, Sweetlove LJ (2010) ROS signaling_specificity is required. Trends Plant Sci 15: 370-374

Mueller S, Hilbert B, Dueckershoff K, Roitsch T, Krischke M, Mueller MJ, Berger S (2008) General detoxification and stress responses are mediated by oxidized lipids through TGA transcription factors in Arabidopsis. Plant Cell 20: 768-785

Pötter W, Karst U (1996) Identification of chemical interferences in aldehyde and ketone determination using dual-wavelength detection. Anal Chem 68: 3354-3358

Reynolds T (1977) Comparative effects of aliphatic compounds on inhibition of lettuce fruit germination. Ann Bot (Lond) 41: 637-648

Takami T, Shibata M, Kobayashi Y, Shikanai T (2010) De novo biosynthesis of fatty acids plays critical roles in the responses of the photosynthetic machinery to low temperature in
Arabidopsis. Plant Cell Physiol 51: 1265-1275

Taki N, Sasaki-Sekimoto Y, Obayashi T, Kikuta A, Kobayashi K, Ainai T, Yagi K, Sakurai N, Suzuki H, Masuda T, et al. (2005) 12-Oxo-phytodienoic acid triggers expression of a distinct set of genes and plays a role in wound-induced gene expression in arabidopsis. Plant Physiol 139: 1268-1283

Triantaphylidès C, Krischke M, Hoeberichts FA, Ksas B, Gresser G, Havaux M, Van Breusegem F, Mueller MJ (2008) Singlet oxygen is the major reactive oxygen species involved in photooxidative damage to plants. Plant Physiol 148: 960-968

Weber H, Chételat A, Reymond P, Farmer EE (2004) Selective and powerful stress gene expression in Arabidopsis in response to malondialdehyde. Plant J 37: 877-888

West JD, Marnett LJ (2006) Endogenous reactive intermediates as modulators of cell signaling and cell death. Chem Res Toxicol 19: 173-194

Yamauchi Y, Hasegawa A, Mizutani M, Sugimoto Y (2012) Chloroplastic NADPH-dependent alkenal/one oxidoreductase contributes to the detoxification of reactive carbonyls produced under oxidative stress. FEBS Lett 586: 1208-1213

Yin L, Mano J, Wang S, Tsuji W, Tanaka K (2010) The involvement of lipid peroxide-derived aldehydes in aluminum toxicity of tobacco roots. Plant Physiol 152: 1406-1417

Zoeller M, Singl N, Krischke M, Fekete A, Waller F, Berger S, Mueller MJ (2012) Lipid profiling of the Arabidopsis hypersensitive response reveals specific lipid peroxidation and fragmentation processes: Biogenesis of pimelic and azelaic acid. Plant Physiol 160: 365-378

Zweiner C, Glauner T, Frimmel FH (2002) Method optimization for the determination of carbonyl compounds in disinfected water by DNPH derivatization and LC-ESI-MS-MS. Anal Bioanal Chem 372: 615-621 